Sylwia Szablewska ${ }^{1}$, Zofia Roszkowska², Katarzyna Białożyk-Mularska', Marzena Anna Lewandowska ${ }^{3}$, Krzysztof Roszkowski ${ }^{1}$

${ }^{1}$ Department of Oncology, Radiotherapy and Gynecologic Oncology, Faculty of Health Sciences, Collegium Medicum, Nicolaus Copernicus University, Bydgoszcz, Poland

2Jagiellonian University, Collegium Medicum, Faculty of Medicine, Krakow, Poland

${ }^{3}$ Department of Thoracic Surgery and Tumors, Faculty of Medicine, Collegium Medicum Bydgoszcz, Nicolaus Copernicus University in Torun, Poland

\title{
Long-term survival in patients with NSCLC treated with single-fraction vs. multi-fraction palliative radiotherapy in the case of lung tumour, brain metastases and bone metastases
}

\author{
Corresponding author: \\ Krzysztof Roszkowski \\ Department of Oncology, \\ Radiotherapy and Gynecologic \\ Oncology, Collegium Medicum \\ Nicolaus Copernicus University, \\ Bydgoszcz 85-796, Romanowskiej 2: \\ Poland, tel. +48523743744 \\ e-mail: roszkowskik@cm.umk.p
}

Medical Research Journal 2019; Volume 4, Number 1, 8-12 DOI: 10.5603/MRJ.a2019.0007 Copyright (C) 2019 Via Medica ISSN 2451-2591

\begin{abstract}
Background: Patients with advanced non-small cell lung cancer (NSCLC) are candidates for different types of treatment, including chemotherapy and radiotherapy or supportive care. Despite the fatal prognosis in advanced disease, many experienced radiation oncologists will apply radiation at low doses with the intention of palliative care.

Methods: We used an extensive database of medical patients diagnosed with NSCLC, treated with palliative radiotherapy at the Oncology Centre in Bydgoszcz, from June 1998 to December 2013. A group of 3202 patients was divided into subgroups: Group A)1762 patients irradiated on the lung tumor (without distant metastases): Total dose: A1) 6Gy/1 fr. ( $n=19) ; A 2) 8 G y / 1 f r . ~(n=276) ; A 3) 20 G y / 5 f r . ~(n=1349) ; A 4)$ 30Gy/10fr. $(n=118)$. Group B) 548 patients irradiated on the central nervous system (CNS) metastases: B1) 20Gy/5fr. ( $n=476)$; B2) 30Gy/10fr. $(n=72)$. Group C) 892 patients irradiated on the bone metastases: C1) 8 Gy/1fr. ( $n=452)$; C2) 10Gy/1fr. $(n=30)$; C3) 20Gy/5fr. $(n=341)$; C4) 30Gy/10fr. $(n=69)$.

Results: Patients with irradiation of a lung tumour: The longest OS was observed in the group of patients irradiated with doses of 20 Gy (76\%) and 30 Gy (7\%). Patients with irradiation of bone metastases: No significant differences in OS were observed between the employed fractionation regimens. Patients with irradiation of CNS metastases: The choice of a higher dose of radiation therapy did not demonstrate differences in median OS values compared to a lower dose.

Conclusions: The patients who were prescribed single fraction palliative radiotherapy did not have poorer prognoses or experience shorter survival than patients who were prescribed multi-fraction pRT in the case of lung tumour, brain metastases and bone metastases.

Key words: palliative radiotherapy, survival, NSCLC, single-fraction, multi-fraction
\end{abstract}

Med Res J 2019; 4 (1): 8-12

\section{INTRODUCTION}

Non-small cell lung carcinoma (NSCLC) represents approx. $85 \%$ of cases of lung cancer, with more than half of the patients having distant metastases at diagnosis [1]. Patients with advanced NSCLC are candidates for various therapies, including chemotherapy [2-3], radiation therapy [4-5] and life-supporting therapy [6]. Despite the fatal prognosis in advanced disease, many experienced oncologists will apply low-dose radiation therapy with palliative intent. The choice of an effective radiation dose that will not cause significant complications remains discussible.

In our study, we conducted a retrospective analysis of the effectiveness of palliative radiation therapy in 3202 patients with advanced NSCLC using different methods of radiation dose fractionation depending on the location of lesions (lung tumour, bone metastases, CNS metastases). 
Table 1. Patient characteristics

\begin{tabular}{|c|c|c|c|c|}
\hline & \multicolumn{4}{|c|}{ Location of lesions } \\
\hline & $\begin{array}{l}\text { Number of patients } \\
(\mathrm{n}=3202)\end{array}$ & Lung & Bone & CNS \\
\hline Mean age & $62.54(22-93)$ & $64.93(22-93)$ & $60.04(30-89)$ & $60.65(36-87)$ \\
\hline Sex (male/female) & $2244 / 958$ & $1300 / 462$ & $596 / 296$ & $348 / 200$ \\
\hline Location of lesions & $\begin{array}{l}\text { Number } \\
\text { of patients }\end{array}$ & & & \\
\hline \multicolumn{5}{|l|}{ Lung } \\
\hline 6 Gy & $19(1 \%)$ & & & \\
\hline 8 Gy & $276(16 \%)$ & & & \\
\hline 10 Gy & 0 & & & \\
\hline 20 Gy & 1349 (76\%) & & & \\
\hline 30 Gy & $118(7 \%)$ & & & \\
\hline \multicolumn{5}{|l|}{ Bone } \\
\hline 6 Gy & 0 & & & \\
\hline 8 Gy & $452(51 \%)$ & & & \\
\hline 10 Gy & $30(3 \%)$ & & & \\
\hline 20 Gy & $341(38 \%)$ & & & \\
\hline 30 Gy & $69(8 \%)$ & & & \\
\hline \multicolumn{5}{|l|}{ CNS } \\
\hline 6 Gy & 0 & & & \\
\hline 8 Gy & 0 & & & \\
\hline 10 Gy & 0 & & & \\
\hline 20 Gy & $476(87 \%)$ & & & \\
\hline 30 Gy & $72(13 \%)$ & & & \\
\hline
\end{tabular}

\section{Material and methods}

\section{Patient database}

An extensive medical database of patients treated at the Oncology Center in Bydgoszcz, Poland, was employed. The analysis included patients diagnosed with advanced NSCLC with no prior surgical intervention, treated with palliative radiation therapy between June 1998 and December 2013.

A group of 3202 patients was divided into three subgroups:

A) 1762 patients subjected to primary irradiation of a lung tumour (with no distant metastases revealed in imaging)

A-1) within this group, 658 patients received chemotherapy after radiation therapy

B) 548 patients subjected to primary irradiation of brain metastases

C) 892 patients subjected to irradiation of bone metastases

Data collected from all patients included: demographic data, symptoms, initial disease stage, prog- nostic factors, including weight loss and fitness, and therapeutic parameters of irradiation, including radiation dose, fractionation and total treatment time. Date of death was used as the data cut-off point. Every patient expressed and signed their informed consent to therapy with palliative intent. Detailed patient characteristics are presented in Table 1.

\section{Treatment methods}

Palliative radiation therapy was administered to all studied patients with non-small cell lung carcinoma. The areas of irradiation included lung tumour, metastatic bone tumour and metastases to the CNS. Doses ranging from 6 Gy to 30 Gy were administered with a different fractionation regimen (1-10 fractions) and total treatment time (1-10 days). Systemic treatment was offered to all patients with stage IV NSCLC.

The total number of patients undergoing palliative radiation therapy was 3202, including 1762 patients with irradiation of a lung tumour (without distant metastases), 548 patients with irradiation of brain metastases and 892 patients with irradiation of bone metastases. 
Table 2. OS values for the three subgroups of patients depending on the dose of radiation therapy and the location of the treated metastases

\begin{tabular}{|c|c|c|c|c|}
\hline \multicolumn{5}{|c|}{ Lung } \\
\hline Dose/Number of patients & $\begin{array}{c}6 \text { Gy } \\
(n=19)\end{array}$ & $\begin{array}{c}8 \text { Gy } \\
(n=276)\end{array}$ & $\begin{array}{c}20 \text { Gy } \\
(n=1349)\end{array}$ & $\begin{array}{c}30 \mathrm{~Gy} \\
(n=118)\end{array}$ \\
\hline Median OS (months) & 6 & 5 & 7 & 7 \\
\hline \multicolumn{5}{|c|}{ Bone } \\
\hline Dose/Number of patients & $\begin{array}{c}8 \text { Gy } \\
(n=452)\end{array}$ & $\begin{array}{c}10 \text { Gy } \\
(n=30)\end{array}$ & $\begin{array}{c}20 \text { Gy } \\
(n=341)\end{array}$ & $\begin{array}{c}30 \text { Gy } \\
(n=69)\end{array}$ \\
\hline Median OS (months) & 5 & 4 & 4 & 5 \\
\hline \multicolumn{5}{|c|}{ CNS } \\
\hline Dose/Number of patients & - & - & $\begin{array}{c}20 \text { Gy } \\
(n=476)\end{array}$ & $\begin{array}{c}30 \text { Gy } \\
(n=72)\end{array}$ \\
\hline Median OS (months) & - & - & 4 & 4 \\
\hline
\end{tabular}

After the completion of radiation therapy, $100 \%$ of the treated patients of each group were followed-up for overall survival (OS). The three subgroups of patients were irradiated with different doses, which constituted a criterion of patient selection and follow-up for OS in these subgroups (Table 1).

\section{Statistical analysis}

The arithmetic mean and median OS was calculated using the Microsoft ${ }^{\circledR}$ Excel 2011 spreadsheet. Statistical significance of differences between the obtained results was checked using the logrank test $(p<0.05)$ in the STATISTICA software v. 13.0 by StatSoft. Comparative analysis of OS for all investigated groups was conducted using the Kaplan-Meyer method.

\section{Results}

\section{Patients with irradiation of a lung tumour:}

Median OS was 5-7 months after the completion of radiation therapy. The longest OS was observed in the group of patients irradiated with doses of $20 \mathrm{~Gy}(76 \%)$ and 30 Gy (7\%). Patients who received lower single doses of 6 Gy (1\%) and 8 Gy (16\%) survived an insignificantly shorter period, but this could be caused by the small size of the group and the patients' individual disease characteristics (logrank test, $p=0.06$ ) (Table 2 , Fig. 1).

\section{Patients with irradiation of bone metastases:}

No significant differences in OS were observed between the employed fractionation regimens (Table 2, Fig. 2). The choice of a single radiation fraction for bone metastases not only resulted in a similar OS as with

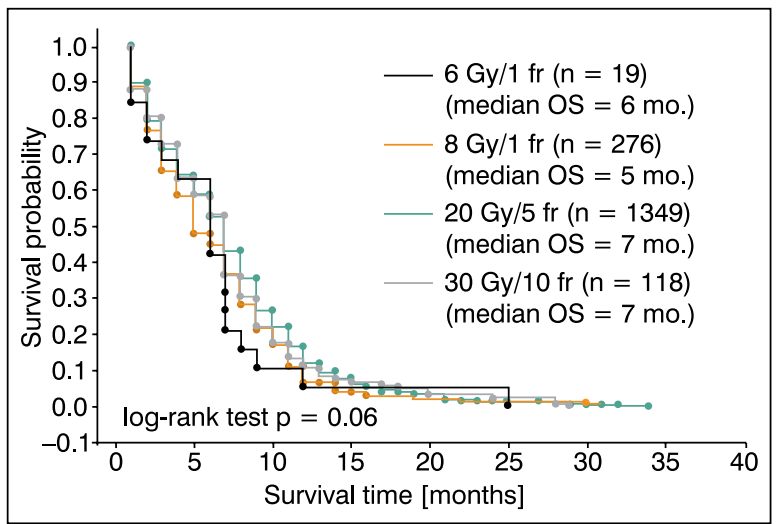

Figure 1. Kaplan-Meier survival curves for patients diagnosed with a lung tumor treated with palliative radiation therapy at different doses. Curves for each radiation dose have a very similar shape and course. The course is almost identical in the first 4 months and after the 14th month (most deaths occurred before the 14th month following the completion of radiation therapy). The small visible increase in the number of deaths of patients irradiated with the $6 \mathrm{~Gy}$ dose can be a result of a statistical error, as the group of patients irradiated with the 6 Gy dose is clearly smaller than the other groups. Individual patient characteristics could contribute to the results obtained in this small subgroup as well. In the 6th month, a large increase in the number of deaths in the group irradiated with the 30 Gy dose can be seen. The graphs for patients irradiated with the 8 and 20 Gy doses are very similar throughout their continuity, with minor deviations seen only between the 5th and the 6th month. Differences in the OS values for each subgroup of patients are statistically insignificant (logrank test, $p=0.06)$.

a higher dose administered in a few fractions but also allowed one-day treatment.

\section{Patients with irradiation of CNS metastases:}

The choice of a higher dose of radiation therapy did not demonstrate differences in median OS values 


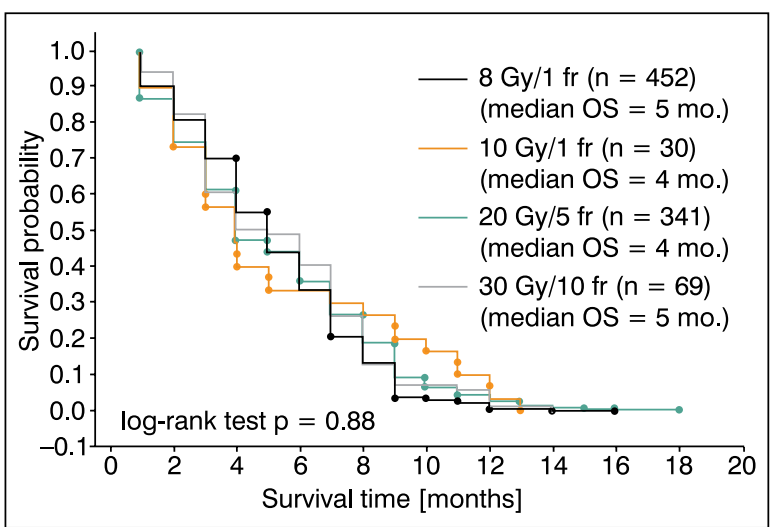

Figure 2. Kaplan-Meier survival curves for patients diagnosed with bone metastases of NSCLC treated with palliative radiation therapy at different doses. The course of four curves is comparable and illustrates the lack of a significant impact of dose level and fractionation on OS (logrank test, $p=0.88$ ). Patients irradiated with the $10 \mathrm{~Gy}$ dose after the 4th month have a more flat curve than the other doses, with a proportionally lower death rate (a small number of deaths between the 4th and the 9th month). In the group of patients who received the $30 \mathrm{~Gy}$ dose, a sudden increase in the number of deaths in the 3rd month can be seen. Curves for the 8 and 20 Gy doses, the most commonly used doses in clinical practice, have a very similar course with minor deviations in each month. The similar course of curves for the groups irradiated with the 8 Gy dose in 1 fraction and the 20 Gy dose in 5 fractions (OS $=5$ months) should lead to the choice of irradiation in a single dose in these clinical cases.

compared to a lower dose. Based on the conducted analyses, it was found that increasing the total radiation dose does not extend the mean OS values (Table 2, Fig. 3).

\section{Discussion}

Palliative radiation therapy administered in a single dose allows quickly obtaining the desired effect without exposing patients to prolonged therapeutic procedures. Among the most common benefits of a single administration of a lower dose of radiation therapy in palliative treatment is its analgesic effect [7-8]. Often, bleeding from the upper respiratory ways in cases of tumours located in the lungs is stopped. The most frequent reason for palliative radiation therapy in patients with NSCLC is hemoptysis that can be stopped with low doses administered at appropriate intervals [9].

In a study by Ma et al., a meta-analysis of randomized, controlled trials involving a total of 1730 patients with locally advanced lung cancer was conducted [10]. The aim of this meta-analysis was to compare a higher ( $\geq 30 \mathrm{~Gy}$ ) and lower (<30 Gy) dose of radiation ther-

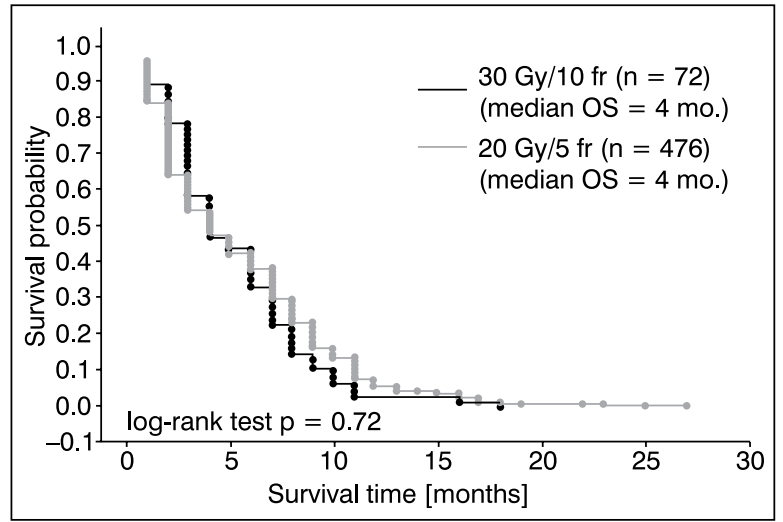

Figure 3. Kaplan-Meier survival curves for patients diagnosed with CNS metastases of NSCLC treated with palliative radiation therapy at different doses. Curves for both doses are very similar. In both cases, most deaths occurred in the first 4 months. In the subsequent months of follow-up, the patients' OS was similar (logrank test, $\mathrm{p}=0.72)$.

apy in mitigating the symptoms and improving patient survival. The combined odds ratios (ORs) did not indicate a significant difference in the mitigation of cough, chest pain and hemoptysis between groups subjected to radiation therapy at the higher and the lower dose. OS values after the 1st and the 2nd year were similar between the high-dose and low-dose groups. In another retrospective study [11] involving 232 patients with NSCLC, the most frequently recommended dose was 2 fractions of 8.5 Gy (34\%), followed by 10 fractions of 3 Gy or equivalent doses (30\%, EQD2 approx. $33 \mathrm{~Gy}$ ). OS was significantly shorter in the case of the $2 \times 8.5$ Gy regimen (median OS 2.5 months compared to 5.0 months for the 33 Gy regimen). In our study, a greater range of therapeutic doses was used, but comparative analysis revealed similar conclusions regarding the benefits of lower doses of radiation therapy in the palliative treatment of locally advanced lung cancer.

In the investigated subgroup of patients with irradiation of bone metastases, no significant differences in OS were found. The choice of a single irradiation with a dose of 8 Gy in 1 fraction for bone metastases not only offers a similar OS, but also allows for further sessions of radiation therapy for the treatment of recurrent osteoarticular pain or improvement of neurological signs in cases of spinal cord compression, as well as for an effective reduction of symptoms caused by compression of neighboring organs by the tumor, bleeding or infiltration [12-16].

The acknowledged regimen for treating CNS metastases is radiation therapy at 30 Gy in 10 fractions. In patients with poor prognosis, a smaller total dose of 20 Gy in 5 fractions is used [17]. 
In our study, the choice of the higher dose of radiation therapy did not demonstrate differences in OS compared to the lower dose (Fig. 3). Some researchers pointed out the doubtful results of reduced neurological symptoms after palliative radiation therapy of brain metastases [18-20]. However, a better understanding of both the acute and the chronic toxicity of whole-brain radiation therapy should lead to more selective use of this procedure.

\section{Conclusions}

The use of a single fraction of palliative radiation therapy allows patients to conduct a single comprehensive hospital visit and obtain a therapeutic and analgesic effect, as well as to optimize their quality of life without the need for further hospital visits. In relation to the studied groups, the analysis should include the treatment cost and travel expenses that are lower with a single hospital visit. Therefore, due to the specificity of patients in palliative care and the progression of the disease, the selected therapeutic regimens should be as short as possible but effective, along with a rationally selected radiation dose.

The study demonstrated that the clinical effect of using high-dose irradiation is comparable to that of multi-fraction radiation therapy in palliative treatment.

\section{Disclosure of interest}

The authors declare that they have no conflict of interest

\section{Financial disclosures}

No financial disclosures from any author

\section{References}

1. Wang J, Ji Z, Wang X, et al. Radical thoracic radiotherapy may provide favorable outcomes for stage IV non-small cell lung cancer. Thorac Cancer. 2016; 7(2): 182-189, doi: 10.1111/1759-7714.12305, indexed in Pubmed: 27042220.

2. Cullen MH, Billingham LJ, Woodroffe CM, et al. Mitomycin, ifosfamide, and cisplatin in unresectable non-small-cell lung cancer: effects on survival and quality of life. J Clin Oncol. 1999; 17(10): 3188-3194 doi: 10.1200/JCO.1999.17.10.3188, indexed in Pubmed: 10506617.
3. Shepherd FA. Chemotherapy for non-small cell lung cancer: have we reached a new plateau? Semin Oncol. 1999; 26(1 Suppl 4): 3-11, indexed in Pubmed: 10201515

4. Macbeth FR, Bolger JJ, Hopwood P, et al. Randomized trial of palliative two-fraction versus more intensive 13-fraction radiotherapy for patients with inoperable non-small cell lung cancer and good performance status. Medical Research Council Lung Cancer Working Party. Clin Oncol (R Coll Radiol). 1996; 8(3): 167-175, indexed in Pubmed: 8814371.

5. Tang JI, Shakespeare TP, Lu JJ, et al. Patients' preference for radiotherapy fractionation schedule in the palliation of symptomatic unresectable lung cancer. J Med Imaging Radiat Oncol. 2008; 52(5): 497-502, doi: 10.1111/j.1440-1673.2008.02002.x, indexed in Pubmed: 19032397.

6. Bayly JL, Lloyd-Williams M. Identifying functional impairment and rehabilitation needs in patients newly diagnosed with inoperable lung cancer: a structured literature review. Support Care Cancer. 2016; 24(5): 2359-2379, doi: 10.1007/s00520-015-3066-1, indexed in Pubmed: 26746210

7. Culleton S, Kwok S, Chow E. Radiotherapy for pain. Clin Oncol (R Coll Radiol). 2011; 23(6): 399-406, doi: 10.1016/j.clon.2010.11.011, indexed in Pubmed: 21168999

8. Wu JSY, Wong R, Johnston M, et al. Cancer Care Ontario Practice Guidelines Initiative Supportive Care Group. Meta-analysis of dose-fractionation radiotherapy trials for the palliation of painful bone metastases. Int J Radiat Oncol Biol Phys. 2003; 55(3): 594-605, doi: 10.1016/s0360-3016(02)04147-0, indexed in Pubmed: 12573746.

9. Adamietz I. Radiotherapy. Frontiers of Radiation Therapy and Oncology. 2009: 164-172, doi: 10.1159/000262472.

10. Ma JT, Zheng JH, Han CB, et al. Meta-analysis comparing higher and lower dose radiotherapy for palliation in locally advanced lung cancer. Cancer Sci. 2014; 105(8): 1015-1022, doi: 10.1111/cas.12466, indexed in Pubmed: 24974909

11. Nieder C, Tollali T, Yobuta R, et al. Palliative Thoracic Radiotherapy for Lung Cancer: What Is the Impact of Total Radiation Dose on Survival? J Clin Med Res. 2017; 9(6): 482-487, doi: 10.14740/jocmr2980w, indexed in Pubmed: 28496548.

12. Chow R, Hoskin P, Hollenberg D, et al. Efficacy of single fraction conventional radiation therapy for painful uncomplicated bone metastases: a systematic review and meta-analysis. Ann Palliat Med. 2017; 6(2): 125-142, doi: 10.21037/apm.2016.12.04, indexed in Pubmed: 28249544

13. Bedard G, Hoskin P, Chow E. Overall response rates to radiation therapy for patients with painful uncomplicated bone metastases undergoing initial treatment and retreatment. Radiother Oncol. 2014; 112(1): 125-127, doi: 10.1016/j.radonc.2014.06.015, indexed in Pubmed: 25023043

14. Schofield $P$, Ball D, Smith JG, et al. Optimism and survival in lung carcinoma patients. Cancer. 2004; 100(6): 1276-1282, doi: 10.1002/cncr.20076, indexed in Pubmed: 15022297.

15. Mott TF. Lung Cancer: Management. FP Essent. 2018; 464: 27-30, indexed in Pubmed: 29313655.

16. Frank MS, Nørøxe DS, Nygård L, et al. Fractionated palliative thoracic radiotherapy in non-small cell lung cancer - futile or worth-while? BMC Palliative Care. 2018; 17(1), doi: 10.1186/s12904-017-0270-4.

17. Wang TJC, Brown PD. Brain metastases: fractionated whole-brain radiotherapy. Handb Clin Neurol. 2018; 149: 123-127, doi: 10.1016/B9780-12-811161-1.00009-8, indexed in Pubmed: 29307349.

18. Miyazawa K, Shikama N, Okazaki S, et al. Predicting prognosis of short survival time after palliative whole-brain radiotherapy. J Radiat Res. 2018; 59(1): 43-49, doi: 10.1093/jrr/rrx058, indexed in Pubmed: 29069502

19. Tsakonas G, Hellman F, Gubanski M, et al. Prognostic factors affecting survival after whole brain radiotherapy in patients with brain metastasized lung cancer. Acta Oncol. 2018; 57(2): 231-238, doi: 10.1080/0 284186X.2017.1386799, indexed in Pubmed: 28984492

20. Nieder C, Norum J, Hintz M, et al. Short Survival Time after Palliative whole Brain Radiotherapy: Can We Predict Potential Overtreatment by Use of a Nomogram? J Cancer. 2017; 8(9): 1525-1529, doi: 10.7150/jca.18600, indexed in Pubmed: 28775771. 